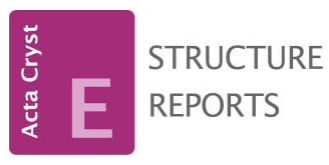

ISSN 1600-5368

Received 9 November 2014

Accepted 12 November 2014

Edited by H. Stoeckli-Evans, University of Neuchâtel, Switzerland

Keywords: crystal structure; phenoxyacetic acid salts; MCPA; herbicides; ammonium carboxylates; hydrogen bonding

CCDC references: 1033945; 1033946 ; 1033947

Supporting information: this article has supporting information at journals.iucr.org/e

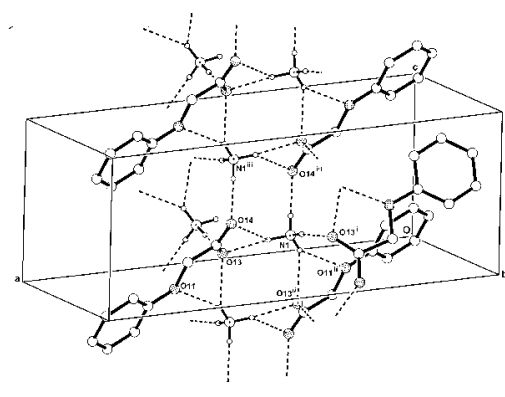

OPEN $\odot$ ACCESS

\section{Two-dimensional hydrogen-bonded polymers in the crystal structures of the ammonium salts of phenoxyacetic acid, (4-fluorophenoxy)acetic acid and (4-chloro-2-methylphenoxy)acetic acid}

\author{
Graham Smith
}

Science and Engineering Faculty, Queensland University of Technology, GPO Box 2434, Brisbane, Queensland 4001, Australia. *Correspondence e-mail: g.smith@qut.edu.au

The structures of the ammonium salts of phenoxyacetic acid, $\mathrm{NH}_{4}{ }^{+} \cdot \mathrm{C}_{8} \mathrm{H}_{6} \mathrm{O}_{3}{ }^{-}$, (I), (4-fluorophenoxy)acetic acid, $\mathrm{NH}_{4}{ }^{+} \cdot \mathrm{C}_{8} \mathrm{H}_{5} \mathrm{FO}_{3}{ }^{-}$, (II), and the herbicidally active (4-chloro-2-methylphenoxy)acetic acid (MCPA), $\mathrm{NH}_{4}{ }^{+} \cdot \mathrm{C}_{9} \mathrm{H}_{8} \mathrm{ClO}_{3}{ }^{-}$.$0.5 \mathrm{H}_{2} \mathrm{O}$, (III) have been determined. All have two-dimensional layered structures based on inter-species ammonium $\mathrm{N}-\mathrm{H} \cdots \mathrm{O}$ hydrogen-bonding associations, which give core substructures consisting primarily of conjoined cyclic motifs. The crystals of (I) and (II) are isomorphous with the core comprising $R_{1}^{2}(5), R_{1}^{2}(4)$ and centrosymmetric $R_{4}^{2}(8)$ ring motifs, giving twodimensional layers lying parallel to (100). In (III), the water molecule of solvation lies on a crystallographic twofold rotation axis and bridges two carboxyl $\mathrm{O}$ atoms in an $R_{4}^{4}(12)$ hydrogen-bonded motif, creating two $R_{4}^{3}(10)$ rings, which together with a conjoined centrosymmetric $R_{4}^{2}(8)$ ring incorporating both ammonium cations, generate two-dimensional layers lying parallel to (100). No $\pi-\pi$ ring associations are present in any of the structures.

\section{Chemical context}

The crystal structures of the ammonium salts of carboxylic acids are, despite their simple formulae, characterized by the presence of a complex array of hydrogen-bonding interactions. From a study of the packing motifs of the these ammonium carboxylate salts from examples in the Cambridge Structural Database (Groom \& Allen, 2014), Odendal et al. (2010) found that two-dimensional hydrogen-bonded nets, ladders or cubane-type structures could be predicted on the basis of the size and conformation of the anions. These structures are often stabilized by $\pi-\pi$ aromatic ring interactions. With the benzoic acid analogues, two-dimensional sheet structures are common with interactions involving the ammonium cations and the carboxylate anions in $\mathrm{N}-\mathrm{H} \cdots \mathrm{O}$ hydrogen bonding, forming core layer structures, with the aromatic rings occupying the interstitial cell regions, e.g. with benzoic acid (Odendal et al., 2010), 3-nitrobenzoic acid (Eppel \& Bernstein, 2009) and 2,4-dichlorobenzoic acid (Smith, 2014). Three-dimensional structures are usually only formed when interactive substituent groups are present on the benzoate rings, interlinking the layers e.g. with 3,5-dinitrobenzoic acid (Smith, 2014). The presence of water molecules of solvation may also produce a similar effect, although these are usually confined to the primary cation-anion layers.

With the phenoxyacetic acid analogues, which comprise a number of herbicidally active commercial herbicides 
(Zumdahl, 2010), this should also be the case. In the only reported structure of an ammonium salt of a phenoxyacetic acid [with the commercially important herbicide, the 2,4-dichloro-substituted analogue (2,4-D) (a hemihydrate) (Liu et al., 2009)], the expected two-dimensional layered structure is found. Herein are reported the preparation and structures of the anhydrous ammonium salts of the parent phenoxyacetic acid, $\mathrm{NH}_{4}^{+} \cdot \mathrm{C}_{8} \mathrm{H}_{6} \mathrm{O}_{3}{ }^{-}$(I) and (4-fluorophenoxy)acetic acid, $\mathrm{NH}_{4}{ }^{+} \cdot \mathrm{C}_{8} \mathrm{H}_{5} \mathrm{FO}_{3}{ }^{-}$(II) and the hemihydrate salt of the herbicidally active (4-chloro-2-methylphenoxy)acetic acid (MCPA), $\mathrm{NH}_{4}{ }^{+} \cdot \mathrm{C}_{9} \mathrm{H}_{8} \mathrm{ClO}_{3}{ }^{-} \cdot 0.5 \mathrm{H}_{2} \mathrm{O}$ (III). The structure of a hydrated chloromethylammonium salt of MCPA is known (Pernak et al., 2011).
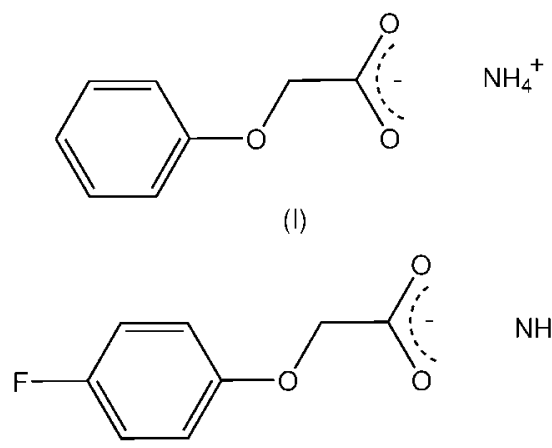

$\mathrm{NH}_{4}^{+}$

(II)

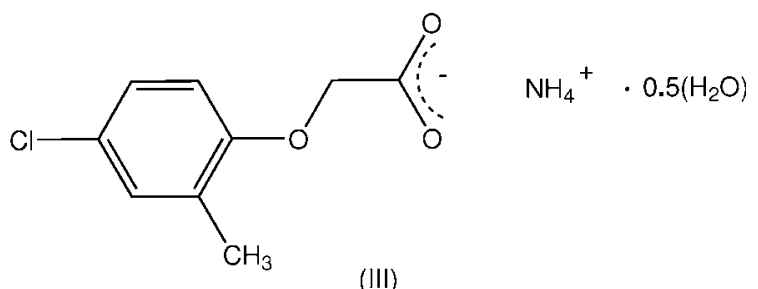

(III)

\section{Structural commentary}

In the structures of the isomorphous ammonium phenoxyacetate (I) and (4-fluorophenoxy)acetate (II) (Figs. 1 and 2, respectively), the anionic species are essentially planar; the comparative defining torsion angles in the phenoxyacetate side chain $(\mathrm{C} 2-\mathrm{C} 1-\mathrm{O} 11-\mathrm{C} 12, \mathrm{C} 1-\mathrm{O} 11-\mathrm{C} 12-\mathrm{C} 13$ and $\mathrm{O} 11-\mathrm{C} 12-\mathrm{C} 13-\mathrm{O} 14)$ are $178.93(19),-177.48(18)$ and $-173.58(18)^{\circ}$, respectively, for (I) and $-179.05(18)$, -178.98 (17) and $-174.13(17)^{\circ}$, respectively, for (II). This planarity is also found in the MCPA anion in (III) (Fig. 3) where the corresponding torsion angles are $-179.13(15)$, -173.34 (14) and $-178.71(15)^{\circ}$ and is also the case with the parent acids [for (I): Kennard et al. (1982), for (II): Smith et al. (1992) and for (III): Smith \& Kennard (1981); Sieron et al. (2011)]. In (III), the water molecule of solvation lies on a crystallographic twofold rotation axis.

\section{Supramolecular features}

In the crystals of (I) and (II), two $\mathrm{H}$ atoms of the ammonium group give cyclic asymmetric three-centre (bifurcated)

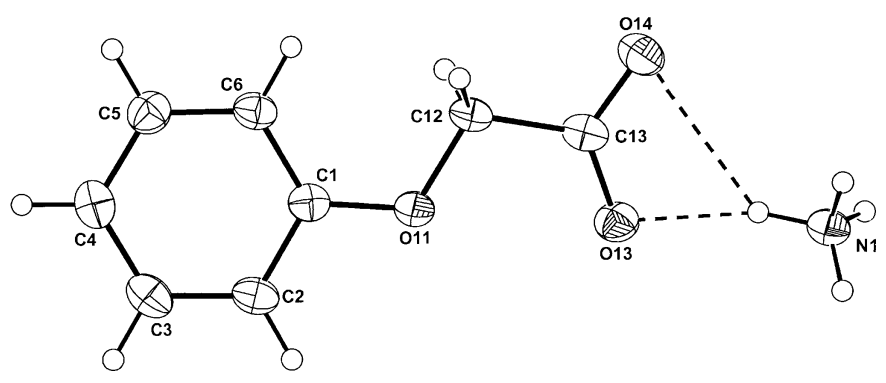

Figure 1

Molecular conformation and atom labelling for (I), with inter-species hydrogen bonds shown as a dashed lines (see Table 1 for details). Non-H atoms are shown as $40 \%$ probability displacement ellipsoids.

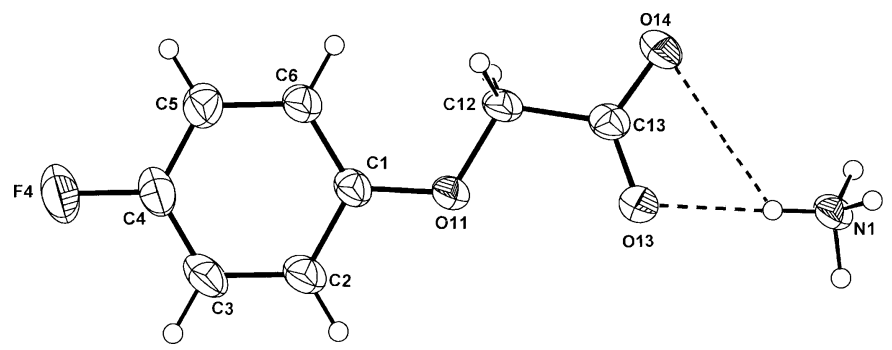

Figure 2

Molecular conformation and atom labelling for (II), with inter-species hydrogen bonds shown as dashed lines (see Table 2 for details). Non-H atoms are shown as $40 \%$ probability displacement ellipsoids.

$\mathrm{N}-\mathrm{H} \cdots(\mathrm{O}, \mathrm{O})$ hydrogen-bonding interactions with the anion (Tables 1 and 2, respectively). One of these is with two O-atom acceptors of the carboxyl group (O13, O14) [graph set $R_{1}^{2}(4)$ ], the other is with the carboxyl and phenoxy O-atom acceptors $\left(\mathrm{O} 13^{\mathrm{ii}}, \mathrm{O} 11^{\mathrm{ii}}\right)$ of an inversion-related anion [graph set $\left.R_{1}^{2}(5)\right]$. These, together with a third $\mathrm{N} 1-\mathrm{H} 13 \cdots \mathrm{O} 13^{\mathrm{ii}}$ hydrogen bond, give a cyclic $R_{4}^{2}(8)$ ring motif, forming a series of conjoined rings which extend the structures along $c$. The other $\mathrm{H}$ atom gives structure extension through an $\mathrm{N}-\mathrm{H} \cdots \mathrm{O}$ hydrogen bond to a carboxyl $\mathrm{O}$ atom $\left(\mathrm{O} 14^{\mathrm{iii}}\right)$, forming a two-dimensional sheet-like structure which lies parallel to (100). Present in the crystal are short inversion-related intermolecular F4 $\cdots \mathrm{F} 4^{\text {iv }}$ contacts of 2.793 (2) $\AA$ [symmetry code: (iv) $-x+2$,

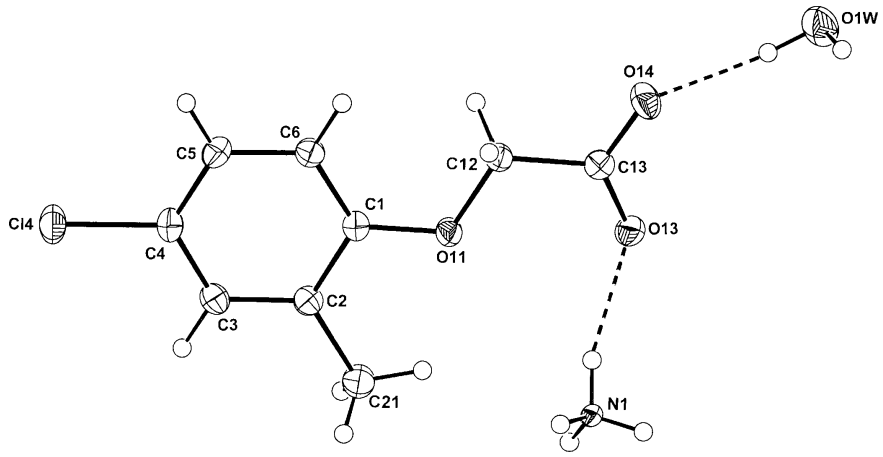

Figure 3

Molecular conformation and atom labelling for (III), with inter-species hydrogen bonds shown as dashed lines (see Table 3 for details). Non-H atoms are shown as $40 \%$ probability displacement ellipsoids. 
Table 1

Hydrogen-bond geometry $\left(\AA{ }^{\circ}\right)$ for $(\mathrm{I})$.

\begin{tabular}{lllll}
\hline$D-\mathrm{H} \cdots A$ & $D-\mathrm{H}$ & $\mathrm{H} \cdots A$ & $D \cdots A$ & $D-\mathrm{H} \cdots A$ \\
\hline $\mathrm{N} 1-\mathrm{H} 11 \cdots \mathrm{O} 13$ & 0.96 & 1.92 & $2.849(3)$ & 163 \\
$\mathrm{~N} 1-\mathrm{H} 11 \cdots \mathrm{O} 14$ & 0.96 & 2.55 & $3.330(3)$ & 138 \\
$\mathrm{~N} 1-\mathrm{H} 12 \cdots \mathrm{O} 13^{\mathrm{i}}$ & 0.85 & 2.03 & $2.867(3)$ & 172 \\
$\mathrm{~N} 1-\mathrm{H} 13 \cdots \mathrm{O} 11^{\mathrm{ii}}$ & 0.90 & 2.39 & $3.202(3)$ & 150 \\
$\mathrm{~N} 1-\mathrm{H} 13 \cdots \mathrm{O} 13^{\mathrm{ii}}$ & 0.90 & 2.15 & $2.869(3)$ & 136 \\
$\mathrm{~N} 1-\mathrm{H} 14 \cdots \mathrm{O} 14^{\mathrm{iii}}$ & 0.84 & 1.95 & $2.788(3)$ & 178 \\
\hline Symmetry codes: (i) & $-x+1, y+\frac{1}{2},-z+\frac{1}{2} ;$ & (ii) $-x+1,-y+1,-z ; \quad$ (iii) \\
$-x+1,-y+1,-z+1$. & & &
\end{tabular}

Table 2

Hydrogen-bond geometry $\left(\AA,^{\circ}\right)$ for (II).

\begin{tabular}{lllll}
\hline$D-\mathrm{H} \cdots A$ & $D-\mathrm{H}$ & $\mathrm{H} \cdots A$ & $D \cdots A$ & $D-\mathrm{H} \cdots A$ \\
\hline $\mathrm{N} 1-\mathrm{H} 11 \cdots \mathrm{O} 13$ & 0.90 & 1.95 & $2.847(2)$ & 177 \\
$\mathrm{~N} 1-\mathrm{H} 11 \cdots \mathrm{O} 14$ & 0.90 & 2.55 & $3.347(2)$ & 135 \\
$\mathrm{~N} 1-\mathrm{H} 12 \cdots \mathrm{O} 13^{\mathrm{i}}$ & 0.97 & 1.88 & $2.847(3)$ & 173 \\
$\mathrm{~N} 1-\mathrm{H} 13 \cdots \mathrm{O} 11^{\mathrm{ii}}$ & 0.96 & 2.36 & $3.172(2)$ & 142 \\
$\mathrm{~N} 1-\mathrm{H} 13 \cdots \mathrm{O} 13^{\mathrm{ii}}$ & 0.96 & 2.13 & $2.892(2)$ & 135 \\
$\mathrm{~N} 1-\mathrm{H} 14 \cdots \mathrm{O} 14^{\mathrm{iii}}$ & 0.89 & 1.91 & $2.793(2)$ & 173 \\
\hline
\end{tabular}

Symmetry codes: (i) $\quad-x+1, y+\frac{1}{2},-z+\frac{1}{2} ; \quad$ (ii) $\quad-x+1,-y+1,-z ; \quad$ (iii) $-x+1,-y+1,-z+1$.

Table 3

Hydrogen-bond geometry $\left(\AA,^{\circ}\right)$ for (III).

\begin{tabular}{|c|c|c|c|c|}
\hline$D-\mathrm{H} \cdots A$ & $D-\mathrm{H}$ & $\mathrm{H} \cdots A$ & $D \cdots A$ & $D-\mathrm{H} \cdots A$ \\
\hline $\mathrm{N} 1-\mathrm{H} 11 \cdots \mathrm{O} 13^{\mathrm{i}}$ & 0.82 & 2.21 & $2.998(4)$ & 161 \\
\hline $\mathrm{N} 1-\mathrm{H} 12 \cdots \mathrm{O} 14^{\mathrm{ii}}$ & 0.82 & 2.09 & $2.886(4)$ & 166 \\
\hline $\mathrm{N} 1-\mathrm{H} 13 \cdots \mathrm{O} 13^{\mathrm{iii}}$ & 0.84 & 2.04 & 2.877 (4) & 173 \\
\hline $\mathrm{N} 1-\mathrm{H} 14 \ldots \mathrm{O} 13$ & 0.82 & 2.00 & $2.798(4)$ & 163 \\
\hline $\mathrm{O} 1 W-\mathrm{H} 11 W \cdots \mathrm{O} 14$ & 0.88 & 1.95 & $2.809(4)$ & 165 \\
\hline
\end{tabular}

Symmetry codes: (i) $-x+1,-y+2,-z+1$; (ii) $x,-y+2, z-\frac{1}{2}$; (iii) $x, y-1, z$.

$-y+1,-z-1]$. The crystal packing and hydrogen-bonding in (I) is identical to that in isostructural (II), as shown in Fig. 4.

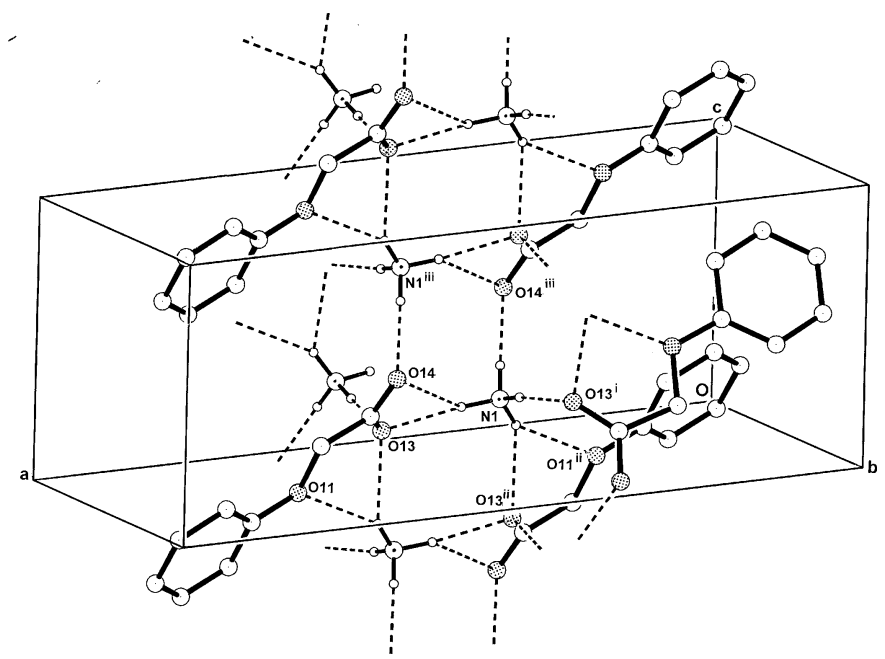

Figure 4

The two-dimensional hydrogen-bonded network structure of (I), which is equivalent to that of the isomorphous compound (II). Hydrogen bonds are shown as dashed lines and non-associative $\mathrm{H}$-atoms have been omitted [for symmetry codes see Tables 1 and 2].

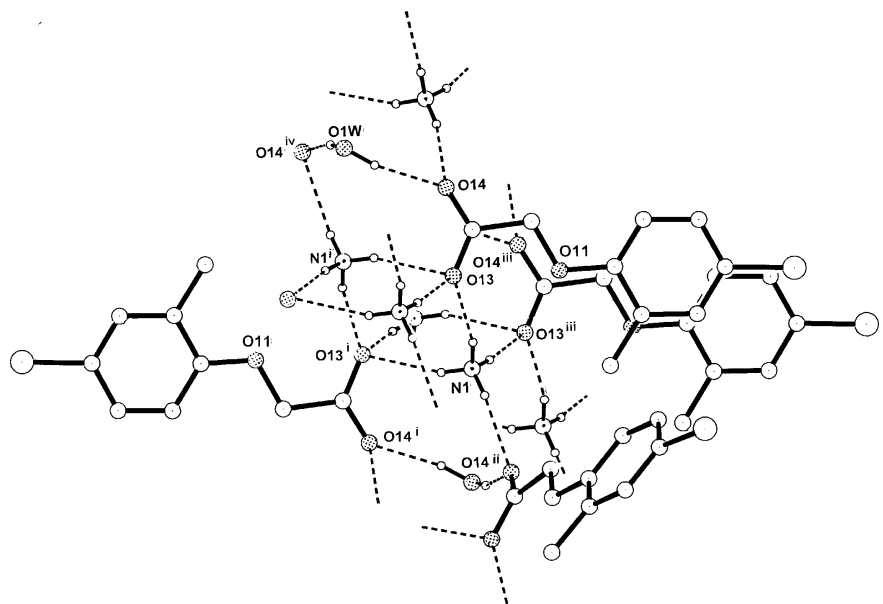

Figure 5

A partial extension of the basic cation-anion hydrogen-bonding associations in the structure of (III), showing conjoined cyclic $R_{4}^{4}(12)$, $R_{4}^{3}(10)$ and $R_{4}^{2}(8)$ ring motifs. [Symmetry code: (iv) $-x+1, y,-z+\frac{3}{2}$. For other codes, see Table 3].

In the crystal of (III), centrosymmetric inter-ion $R_{4}^{2}(8)$ rings are formed between two ammonium cations and two $\mathrm{O} 13$ carboxyl O-atom acceptors and are bridged by a third ammonium $\mathrm{H}$ donor through $\mathrm{O} 13^{\text {iii }}$, extending the structure down $b$ (Table 3 and Fig. 5). The fourth $\mathrm{H}$ atom gives extension along $a$ through $\mathrm{N} 1-\mathrm{H} 12 \cdots \mathrm{O} 14^{\mathrm{ii}}$ forming an enlarged conjoined $R_{4}^{4}(12)$ ring, which is bridged by the water molecule of solvation lying on the twofold rotation axis, through $\mathrm{O} 1 \mathrm{~W}-$ H11W...O14 hydrogen bonds. This link effectively generates two separate $R_{4}^{3}(10)$ ring motifs, extending the structure along $a$ and giving the overall two-dimensional layers lying parallel to (100) (Fig. 6). In (III), no three-centre $R_{1}^{2}(4)$ or $R_{1}^{2}(5)$ motifs to carboxyl $\left(O, O^{\prime}\right)$ or carboxyl-phenoxy $\left(O, O^{1}\right)$ acceptors such as are present in (I) and (II) are found. The structure of (III) is essentially isostructural with that of ammonium (2,4-dichlorophenoxy)acetate hemihydrate (Liu et al., 2009), with isomorphous crystals $[a=37.338(8), b=4.388(9), c=$ 12.900 (3) $\AA, \beta=103.82(3)^{\circ}, V=2074.7$ (8) $\AA^{3}, Z=8$, space group $C 2 / c]$.

No $\pi-\pi$ interactions are found in any of the structures reported here [minimum ring centroid separation = 4.8849 (16) (I), 4.8919 (15) (II) and 4.456 (5) § (III) (the $b$ unit-cell parameter)].

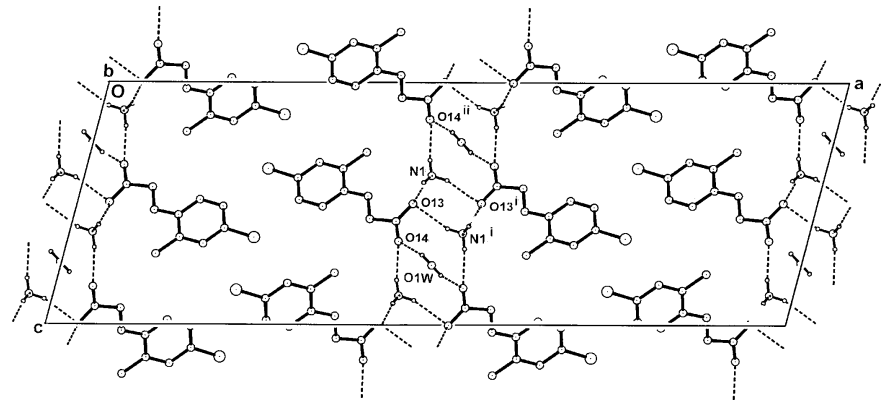

Figure 6

The two-dimensional hydrogen-bonded network structure of (III) in the unit cell, viewed along $b$. 
Table 4

Experimental details.

Crystal data

Chemical formula

$M_{\mathrm{r}}$

Crystal system, space group

Temperature (K)

$a, b, c(\AA)$

$\beta\left({ }^{\circ}\right)$

$V\left(\AA^{3}\right)$

$Z$

Radiation type

$\mu\left(\mathrm{mm}^{-1}\right)$

Crystal size (mm)

Data collection

Diffractometer

Absorption correction

$T_{\min }, T_{\max }$

No. of measured, independent and observed $[I>2 \sigma(I)]$ reflections

$R_{\text {int }}$

$(\sin \theta / \lambda)_{\max }\left(\AA^{-1}\right)$

Refinement

$R\left[F^{2}>2 \sigma\left(F^{2}\right)\right], w R\left(F^{2}\right), S$

No. of reflections

No. of parameters

$\mathrm{H}$-atom treatment

$\Delta \rho_{\max }, \Delta \rho_{\min }\left(\mathrm{e} \AA^{-3}\right)$
(I)

$\mathrm{NH}_{4}{ }^{+} \cdot \mathrm{C}_{8} \mathrm{H}_{7} \mathrm{O}_{3}{ }^{-}$
169.17
Monoclinic, $P 2_{1} / c$
200
$17.824(2), 7.1453(6), 6.7243(7)$
$90.321(9)$
$856.38(15)$
4
Mo $K \alpha$
0.10
$0.35 \times 0.25 \times 0.10$

Oxford Diffraction Gemini-S CCD detector

Multi-scan (CrysAlis PRO;

Agilent, 2013)

$0.920,0.980$

$5450,1686,1218$

0.052

0.617

$\mathrm{NH}_{4}{ }^{+} \cdot \mathrm{C}_{8} \mathrm{H}_{6} \mathrm{FO}_{3}{ }^{-}$
187.17
Monoclinic, $P 2_{1} / c$
200
$18.386(2), 7.1223(6), 6.7609(6)$
$93.399(8)$
$883.79(14)$
4
Mo $K \alpha$
0.12
$0.26 \times 0.20 \times 0.05$

Oxford Diffraction Gemini-S CCD

Multi-scan (CrysAlis PRO;

$5619,1738,1304$

0.033

$0.053,0.116,1.10$

1738

1686

109

$\mathrm{H}$-atom parameters constrained

$0.29,-0.24$
(II) detector

Agilent, 2013)

$0.960,0.980$

0.617

118

(III)

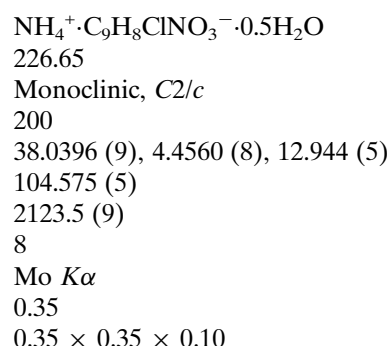

Oxford Diffraction Gemini-S CCD detector

Multi-scan (CrysAlis PRO;

Agilent, 2013)

$0.913,0.980$

$6215,2087,1771$

0.030

0.617

$0.036,0.091,1.03$

2087

132

$\mathrm{H}$-atom parameters constrained $\quad \mathrm{H}$-atom parameters constrained $0.16,-0.22 \quad 0.32,-0.28$

Computer programs: CrysAlis PRO (Agilent, 2013), SIR92 (Altomare et al., 1993), SHELXS97 and SHELXL97 (Sheldrick, 2008) within WinGX (Farrugia, 2012) and PLATON (Spek, 2009).

\section{Synthesis and crystallization}

The title compounds were prepared by the addition of excess $5 \mathrm{M}$ aqueous ammonia solution to $1 \mathrm{mmol}$ of either phenoxyacetic acid [150 mg for (I)], (4-fluorophenoxy)acetic acid [170 mg for (II)] or (4-chloro-2-methylphenoxy)acetic acid [200 mg for (III)] in $10 \mathrm{~mL}$ of $10 \%$ ethanol-water. Roomtemperature evaporation of the solvent gave colourless platelike crystals of (I), (II) and (III) from which specimens were cleaved for the X-ray analyses.

\section{Refinement details}

Crystal data, data collection and structure refinement details are summarized in Table 4. Hydrogen atoms potentially involved in hydrogen-bonding interactions were located in difference Fourier maps but were subsequently included in the refinements with positional parameters fixed and with $U_{\text {iso }}(\mathrm{H})$ $=1.2 U_{\text {eq }}(\mathrm{N})$ or $=1.5 U_{\text {eq }}(\mathrm{O})$. Other $\mathrm{H}$ atoms were included at calculated positions $[\mathrm{C}-\mathrm{H}($ aromatic $)=0.95, \mathrm{C}-\mathrm{H}($ methylene $)=0.98, \mathrm{C}-\mathrm{H}($ methyl $)=0.97 \AA]$ and also treated as riding, with $U_{\text {iso }}(\mathrm{H})=1.5 U_{\text {eq }}(\mathrm{C})$ for methyl $\mathrm{H}$ atoms and $=$ $1.2 U_{\text {eq }}(\mathrm{C})$ for other $\mathrm{H}$ atoms. In (III), the methyl group was found to be rotationally disordered, with the $H$ atoms distributed over six equivalent half-sites, and was treated accordingly.

\section{Acknowledgements}

The author acknowledges financial support from the Science and Engineering Faculty, Queensland University of Technology.

\section{References}

Agilent (2013). CrysAlis PRO. Agilent Technologies Ltd, Yarnton, England.

Altomare, A., Cascarano, G., Giacovazzo, C. \& Guagliardi, A. (1993). J. Appl. Cryst. 26, 343-350.

Eppel, S. \& Bernstein, J. (2009). Cryst. Growth Des. 9, 1683-1691.

Farrugia, L. J. (2012). J. Appl. Cryst. 45, 849-854.

Groom, C. R. \& Allen, F. H. (2014). Angew. Chem. Int. Ed. 53, 662671.

Kennard, C. H. L., Smith, G. \& White, A. H. (1982). Acta Cryst. B38, 868-875.

Liu, H.-L., Guo, S.-H., Li, Y.-Y. \& Jian, F.-F. (2009). Acta Cryst. E65, o1905.

Odendal, J. A., Bruce, J. C., Koch, K. R. \& Haynes, D. A. (2010). CrystEngComm, 12, 2398-2408.

Pernak, J., Syguda, A., Janiszewska, D., Materna, K. \& Praczyk, T. (2011). Tetrahedron, 67, 4838-4844.

Sheldrick, G. M. (2008). Acta Cryst. A64, 112-122.

Sieron, L., Kobylecka, J. \& Turek, A. (2011). Organic Chemistry International, Volume 2011, Article ID 608165, 5 pages. doi: $10.1155 / 2011 / 608165$.

Smith, G. (2014). Acta Cryst. C70, 315-319. 
Smith, G. \& Kennard, C. H. L. (1981). Cryst. Struct. Commun. 10, 295-299.

Smith, G., Lynch, D. E., Sagatys, D. S., Kennard, C. H. L. \& Katekar, G. F. (1992). Aust. J. Chem. 45, 1101-1108.
Spek, A. L. (2009). Acta Cryst. D65, 148-155.

Zumdahl, R. L. (2010). In A History of Weed Science in the United States. New York: Elsevier. 


\section{supporting information}

Acta Cryst. (2014). E70, 528-532 [doi:10.1107/S160053681402488X]

\section{Two-dimensional hydrogen-bonded polymers in the crystal structures of the ammonium salts of phenoxyacetic acid, (4-fluorophenoxy)acetic acid and (4- chloro-2-methylphenoxy)acetic acid}

\section{Graham Smith}

\section{Computing details}

For all compounds, data collection: CrysAlis PRO (Agilent, 2013); cell refinement: CrysAlis PRO (Agilent, 2013); data reduction: CrysAlis PRO (Agilent, 2013). Program(s) used to solve structure: SIR92 (Altomare et al., 1993) for (I); SHELXS97 (Sheldrick, 2008) for (II), (III). For all compounds, program(s) used to refine structure: SHELXL97 (Sheldrick, 2008) within WinGX (Farrugia, 2012); molecular graphics: PLATON (Spek, 2009); software used to prepare material for publication: PLATON (Spek, 2009).

\section{(I) Ammonium phenoxyacetate}

Crystal data

$\mathrm{NH}_{4}^{+} \cdot \mathrm{C}_{8} \mathrm{H}_{7} \mathrm{O}_{3}^{-}$

$M_{r}=169.17$

Monoclinic, $P 2{ }_{1} / c$

Hall symbol: -P 2ybc

$a=17.824(2) \AA$

$b=7.1453(6) \AA$

$c=6.7243(7) \AA$

$\beta=90.321(9)^{\circ}$

$V=856.38(15) \AA^{3}$

$Z=4$

\section{Data collection}

Oxford Diffraction Gemini-S CCD-detector diffractometer

Radiation source: Enhance (Mo) X-ray source

Graphite monochromator

Detector resolution: 16.077 pixels $\mathrm{mm}^{-1}$

$\omega$ scans

Absorption correction: multi-scan

(CrysAlis PRO; Agilent, 2013)

$T_{\min }=0.920, T_{\max }=0.980$

Refinement

Refinement on $F^{2}$

Least-squares matrix: full

$R\left[F^{2}>2 \sigma\left(F^{2}\right)\right]=0.063$

$w R\left(F^{2}\right)=0.163$

$S=1.10$
$F(000)=360$

$D_{\mathrm{x}}=1.312 \mathrm{Mg} \mathrm{m}^{-3}$

Mo $K \alpha$ radiation, $\lambda=0.71073 \AA$

Cell parameters from 1041 reflections

$\theta=3.4-25.5^{\circ}$

$\mu=0.10 \mathrm{~mm}^{-1}$

$T=200 \mathrm{~K}$

Plate, colourless

$0.35 \times 0.25 \times 0.10 \mathrm{~mm}$

5450 measured reflections

1686 independent reflections

1218 reflections with $I>2 \sigma(I)$

$R_{\text {int }}=0.052$

$\theta_{\max }=26.0^{\circ}, \theta_{\min }=3.4^{\circ}$

$h=-21 \rightarrow 21$

$k=-8 \rightarrow 8$

$l=-8 \rightarrow 8$

1686 reflections

109 parameters

0 restraints

Primary atom site location: structure-invariant direct methods 
Secondary atom site location: difference Fourier map

Hydrogen site location: inferred from neighbouring sites

$\mathrm{H}$-atom parameters constrained

$$
\begin{aligned}
& w=1 /\left[\sigma^{2}\left(F_{\mathrm{o}}^{2}\right)+(0.0706 P)^{2}+0.1562 P\right] \\
& \text { where } P=\left(F_{\mathrm{o}}^{2}+2 F_{\mathrm{c}}{ }^{2}\right) / 3 \\
& (\Delta / \sigma)_{\max }<0.001 \\
& \Delta \rho_{\max }=0.29 \mathrm{e} \AA^{-3} \\
& \Delta \rho_{\min }=-0.24 \mathrm{e} \AA^{-3}
\end{aligned}
$$

Special details

Geometry. Bond distances, angles etc. have been calculated using the rounded fractional coordinates. All su's are estimated from the variances of the (full) variance-covariance matrix. The cell e.s.d.'s are taken into account in the estimation of distances, angles and torsion angles

Refinement. Refinement of $F^{2}$ against ALL reflections. The weighted $R$-factor $w R$ and goodness of fit $S$ are based on $F^{2}$,

\begin{tabular}{|c|c|c|c|c|}
\hline & $x$ & $y$ & $z$ & $U_{\text {iso }} * / U_{\text {eq }}$ \\
\hline $\mathrm{O} 11$ & $0.71681(10)$ & $0.4894(2)$ & $-0.0073(2)$ & $0.0357(6)$ \\
\hline $\mathrm{O} 13$ & $0.58441(10)$ & $0.4403(2)$ & 0.1635 & $0.0393(6)$ \\
\hline O14 & $0.60999(11)$ & $0.6471(2)$ & 0.4025 & $0.0444(7)$ \\
\hline $\mathrm{C} 1$ & $0.78672(14)$ & 0.4998 & -0.0880 & $0.0317(8)$ \\
\hline $\mathrm{C} 2$ & $0.79737(16)$ & 0.4028 & $-0.2662(4)$ & $0.0373(9)$ \\
\hline $\mathrm{C} 3$ & $0.86706(17)$ & $0.3999(4)$ & $-0.3531(4)$ & $0.0436(10)$ \\
\hline $\mathrm{C} 4$ & $0.92709(16)$ & $0.4938(4)$ & $-0.2672(4)$ & $0.0429(9)$ \\
\hline $\mathrm{C} 5$ & $0.91570(16)$ & $0.5934(3)$ & $-0.0933(4)$ & $0.0404(9)$ \\
\hline C6 & $0.84634(14)$ & $0.5982(3)$ & -0.0039 & $0.0358(9)$ \\
\hline $\mathrm{C} 12$ & $0.70534(14)$ & 0.5849 & $0.1764(3)$ & $0.0329(8)$ \\
\hline C13 & $0.62684(15)$ & $0.5544(3)$ & 0.2524 & $0.0331(8)$ \\
\hline N1 & $0.43517(12)$ & $0.5486(3)$ & $0.2601(3)$ & $0.0355(7)$ \\
\hline $\mathrm{H} 2$ & 0.75650 & 0.33900 & -0.32710 & $0.0450 *$ \\
\hline H3 & 0.87410 & 0.33280 & -0.47350 & $0.0530^{*}$ \\
\hline $\mathrm{H} 4$ & 0.97530 & 0.48990 & -0.32670 & $0.0520^{*}$ \\
\hline H5 & 0.95640 & 0.65960 & -0.03470 & $0.0480^{*}$ \\
\hline H6 & 0.83930 & 0.66830 & 0.11460 & $0.0430 *$ \\
\hline H121 & 0.71410 & 0.72050 & 0.15720 & $0.0390 *$ \\
\hline H122 & 0.74200 & 0.53900 & 0.27630 & $0.0390 *$ \\
\hline H11 & 0.48820 & 0.53280 & 0.24260 & $0.0430 *$ \\
\hline H12 & 0.43220 & 0.66300 & 0.29220 & $0.0430 *$ \\
\hline H13 & 0.40580 & 0.52630 & 0.15370 & $0.0430 *$ \\
\hline H14 & 0.42230 & 0.48720 & 0.36110 & $0.0430 *$ \\
\hline
\end{tabular}
conventional $R$-factors $R$ are based on $F$, with $F$ set to zero for negative $F^{2}$. The threshold expression of $F^{2}>\sigma\left(F^{2}\right)$ is used only for calculating $R$-factors $(\mathrm{gt})$ etc. and is not relevant to the choice of reflections for refinement. $R$-factors based on $F^{2}$ are statistically about twice as large as those based on $F$, and $R$ - factors based on ALL data will be even larger.

Fractional atomic coordinates and isotropic or equivalent isotropic displacement parameters $\left(\AA^{2}\right)$

Atomic displacement parameters $\left(\AA^{2}\right)$

\begin{tabular}{lllllll}
\hline & $U^{11}$ & $U^{22}$ & $U^{33}$ & $U^{12}$ & $U^{13}$ & $U^{23}$ \\
\hline O11 & $0.0366(11)$ & $0.0331(10)$ & $0.0373(10)$ & $-0.0020(7)$ & $0.0004(8)$ & $-0.0089(7)$ \\
O13 & $0.0446(12)$ & $0.0323(9)$ & $0.0409(10)$ & $-0.0068(8)$ & $0.0011(8)$ & $-0.0014(8)$ \\
O14 & $0.0571(13)$ & $0.0391(11)$ & $0.0370(10)$ & $-0.0059(9)$ & $0.0093(9)$ & $-0.0052(8)$ \\
C1 & $0.0372(15)$ & $0.0228(12)$ & $0.0350(13)$ & $0.0037(10)$ & $-0.0040(11)$ & $0.0015(10)$
\end{tabular}




\begin{tabular}{lllllll} 
C2 & $0.0495(17)$ & $0.0276(13)$ & $0.0348(14)$ & $0.0010(11)$ & $-0.0030(11)$ & $-0.0032(10)$ \\
C3 & $0.057(2)$ & $0.0359(14)$ & $0.0380(15)$ & $0.0056(13)$ & $0.0059(13)$ & $-0.0035(11)$ \\
C4 & $0.0421(18)$ & $0.0421(16)$ & $0.0446(15)$ & $0.0049(12)$ & $0.0084(12)$ & $0.0030(12)$ \\
C5 & $0.0405(17)$ & $0.0339(14)$ & $0.0468(16)$ & $-0.0016(11)$ & $-0.0018(12)$ & $-0.0002(11)$ \\
C6 & $0.0424(17)$ & $0.0296(13)$ & $0.0355(14)$ & $0.0025(10)$ & $-0.0024(11)$ & $-0.0042(10)$ \\
C12 & $0.0410(16)$ & $0.0279(12)$ & $0.0299(13)$ & $0.0008(10)$ & $-0.0024(11)$ & $-0.0049(10)$ \\
C13 & $0.0496(17)$ & $0.0224(12)$ & $0.0273(12)$ & $0.0001(11)$ & $-0.0025(11)$ & $0.0039(10)$ \\
N1 & $0.0434(14)$ & $0.0305(11)$ & $0.0325(11)$ & $-0.0006(9)$ & $-0.0014(9)$ & $-0.0023(9)$ \\
\hline
\end{tabular}

Geometric parameters $\left(\AA,{ }^{\circ}\right)$

\begin{tabular}{|c|c|c|c|}
\hline $\mathrm{O} 11-\mathrm{C} 1$ & $1.364(3)$ & $\mathrm{C} 3-\mathrm{C} 4$ & $1.386(4)$ \\
\hline $\mathrm{O} 11-\mathrm{C} 12$ & $1.427(2)$ & $\mathrm{C} 4-\mathrm{C} 5$ & $1.385(4)$ \\
\hline $\mathrm{O} 13-\mathrm{C} 13$ & $1.261(3)$ & $\mathrm{C} 5-\mathrm{C} 6$ & $1.378(4)$ \\
\hline $\mathrm{O} 14-\mathrm{C} 13$ & $1.245(3)$ & $\mathrm{C} 12-\mathrm{C} 13$ & $1.508(4)$ \\
\hline $\mathrm{N} 1-\mathrm{H} 12$ & 0.8500 & $\mathrm{C} 2-\mathrm{H} 2$ & 0.9500 \\
\hline $\mathrm{N} 1-\mathrm{H} 13$ & 0.9000 & $\mathrm{C} 3-\mathrm{H} 3$ & 0.9500 \\
\hline $\mathrm{N} 1-\mathrm{H} 14$ & 0.8400 & $\mathrm{C} 4-\mathrm{H} 4$ & 0.9500 \\
\hline N1-H11 & 0.9600 & $\mathrm{C} 5-\mathrm{H} 5$ & 0.9500 \\
\hline $\mathrm{C} 1-\mathrm{C} 6$ & $1.392(3)$ & $\mathrm{C} 6-\mathrm{H} 6$ & 0.9500 \\
\hline $\mathrm{C} 1-\mathrm{C} 2$ & $1.398(4)$ & $\mathrm{C} 12-\mathrm{H} 121$ & 0.9900 \\
\hline $\mathrm{C} 2-\mathrm{C} 3$ & $1.376(4)$ & $\mathrm{C} 12-\mathrm{H} 122$ & 0.9900 \\
\hline $\mathrm{C} 1-\mathrm{O} 11-\mathrm{C} 12$ & $117.00(18)$ & $\mathrm{O} 13-\mathrm{C} 13-\mathrm{O} 14$ & $125.6(2)$ \\
\hline $\mathrm{H} 12-\mathrm{N} 1-\mathrm{H} 14$ & 106.00 & $\mathrm{O} 13-\mathrm{C} 13-\mathrm{C} 12$ & $119.21(19)$ \\
\hline $\mathrm{H} 13-\mathrm{N} 1-\mathrm{H} 14$ & 113.00 & $\mathrm{C} 3-\mathrm{C} 2-\mathrm{H} 2$ & 120.00 \\
\hline $\mathrm{H} 11-\mathrm{N} 1-\mathrm{H} 12$ & 102.00 & $\mathrm{C} 1-\mathrm{C} 2-\mathrm{H} 2$ & 120.00 \\
\hline $\mathrm{H} 11-\mathrm{N} 1-\mathrm{H} 13$ & 117.00 & $\mathrm{C} 2-\mathrm{C} 3-\mathrm{H} 3$ & 120.00 \\
\hline $\mathrm{H} 11-\mathrm{N} 1-\mathrm{H} 14$ & 108.00 & $\mathrm{C} 4-\mathrm{C} 3-\mathrm{H} 3$ & 120.00 \\
\hline $\mathrm{H} 12-\mathrm{N} 1-\mathrm{H} 13$ & 110.00 & $\mathrm{C} 5-\mathrm{C} 4-\mathrm{H} 4$ & 121.00 \\
\hline $\mathrm{O} 11-\mathrm{C} 1-\mathrm{C} 6$ & $124.3(2)$ & $\mathrm{C} 3-\mathrm{C} 4-\mathrm{H} 4$ & 121.00 \\
\hline $\mathrm{C} 2-\mathrm{C} 1-\mathrm{C} 6$ & $119.5(2)$ & $\mathrm{C} 6-\mathrm{C} 5-\mathrm{H} 5$ & 119.00 \\
\hline $\mathrm{O} 11-\mathrm{C} 1-\mathrm{C} 2$ & $116.3(2)$ & $\mathrm{C} 4-\mathrm{C} 5-\mathrm{H} 5$ & 119.00 \\
\hline $\mathrm{C} 1-\mathrm{C} 2-\mathrm{C} 3$ & $119.9(2)$ & $\mathrm{C} 1-\mathrm{C} 6-\mathrm{H} 6$ & 120.00 \\
\hline $\mathrm{C} 2-\mathrm{C} 3-\mathrm{C} 4$ & $120.8(3)$ & $\mathrm{C} 5-\mathrm{C} 6-\mathrm{H} 6$ & 120.00 \\
\hline $\mathrm{C} 3-\mathrm{C} 4-\mathrm{C} 5$ & $119.0(3)$ & $\mathrm{H} 121-\mathrm{C} 12-\mathrm{H} 122$ & 108.00 \\
\hline $\mathrm{C} 4-\mathrm{C} 5-\mathrm{C} 6$ & $121.2(2)$ & $\mathrm{O} 11-\mathrm{C} 12-\mathrm{H} 121$ & 109.00 \\
\hline $\mathrm{C} 1-\mathrm{C} 6-\mathrm{C} 5$ & $119.7(2)$ & $\mathrm{O} 11-\mathrm{C} 12-\mathrm{H} 122$ & 109.00 \\
\hline $\mathrm{O} 11-\mathrm{C} 12-\mathrm{C} 13$ & $111.22(18)$ & $\mathrm{C} 13-\mathrm{C} 12-\mathrm{H} 121$ & 109.00 \\
\hline $\mathrm{O} 14-\mathrm{C} 13-\mathrm{C} 12$ & $115.2(2)$ & $\mathrm{C} 13-\mathrm{C} 12-\mathrm{H} 122$ & 109.00 \\
\hline $\mathrm{C} 12-\mathrm{O} 11-\mathrm{C} 1-\mathrm{C} 2$ & $178.93(19)$ & $\mathrm{C} 1-\mathrm{C} 2-\mathrm{C} 3-\mathrm{C} 4$ & $-0.7(4)$ \\
\hline $\mathrm{C} 12-\mathrm{O} 11-\mathrm{C} 1-\mathrm{C} 6$ & $-0.7(3)$ & $\mathrm{C} 2-\mathrm{C} 3-\mathrm{C} 4-\mathrm{C} 5$ & $-0.9(4)$ \\
\hline $\mathrm{C} 1-\mathrm{O} 11-\mathrm{C} 12-\mathrm{C} 13$ & $-177.48(18)$ & $\mathrm{C} 3-\mathrm{C} 4-\mathrm{C} 5-\mathrm{C} 6$ & $0.9(4)$ \\
\hline $\mathrm{O} 11-\mathrm{C} 1-\mathrm{C} 2-\mathrm{C} 3$ & $-177.4(2)$ & $\mathrm{C} 4-\mathrm{C} 5-\mathrm{C} 6-\mathrm{C} 1$ & $0.7(4)$ \\
\hline $\mathrm{C} 6-\mathrm{C} 1-\mathrm{C} 2-\mathrm{C} 3$ & $2.3(4)$ & $\mathrm{O} 11-\mathrm{C} 12-\mathrm{C} 13-\mathrm{O} 13$ & $6.7(3)$ \\
\hline $\mathrm{O} 11-\mathrm{C} 1-\mathrm{C} 6-\mathrm{C} 5$ & $177.3(2)$ & $\mathrm{O} 11-\mathrm{C} 12-\mathrm{C} 13-\mathrm{O} 14$ & $-173.58(18)$ \\
\hline $\mathrm{C} 2-\mathrm{C} 1-\mathrm{C} 6-\mathrm{C} 5$ & $-2.3(3)$ & & \\
\hline
\end{tabular}


Hydrogen-bond geometry $\left(\AA,{ }^{\circ}\right)$

\begin{tabular}{lllll}
\hline$D-\mathrm{H} \cdots A$ & $D-\mathrm{H}$ & $\mathrm{H} \cdots A$ & $D \cdots A$ & $D-\mathrm{H} \cdots A$ \\
\hline $\mathrm{N} 1-\mathrm{H} 11 \cdots \mathrm{O} 13$ & 0.96 & 1.92 & $2.849(3)$ & 163 \\
$\mathrm{~N} 1-\mathrm{H} 11 \cdots \mathrm{O} 14$ & 0.96 & 2.55 & $3.330(3)$ & 138 \\
$\mathrm{~N} 1-\mathrm{H} 12 \cdots \mathrm{O} 13^{\mathrm{i}}$ & 0.85 & 2.03 & $2.867(3)$ & 172 \\
$\mathrm{~N} 1-\mathrm{H} 13 \cdots \mathrm{O} 11^{\mathrm{ii}}$ & 0.90 & 2.39 & $3.202(3)$ & 150 \\
$\mathrm{~N} 1-\mathrm{H} 13 \cdots \mathrm{O} 13^{\mathrm{ii}}$ & 0.90 & 2.15 & $2.869(3)$ & 136 \\
$\mathrm{~N} 1-\mathrm{H} 14 \cdots \mathrm{O} 14^{\mathrm{iii}}$ & 0.84 & 1.95 & $2.788(3)$ & 178 \\
\hline
\end{tabular}

Symmetry codes: (i) $-x+1, y+1 / 2,-z+1 / 2$; (ii) $-x+1,-y+1,-z$; (iii) $-x+1,-y+1,-z+1$.

\section{(II) Ammonium (4-fluorophenoxy)acetate}

Crystal data

$\mathrm{NH}_{4}{ }^{+} \cdot \mathrm{C}_{8} \mathrm{H}_{6} \mathrm{FO}_{3}^{-}$

$M_{r}=187.17$

Monoclinic, $P 2_{1} / c$

Hall symbol: -P 2ybc

$a=18.386(2) \AA$

$b=7.1223(6) \AA$

$c=6.7609(6) \AA$

$\beta=93.399(8)^{\circ}$

$V=883.79(14) \AA^{3}$

$Z=4$

\section{Data collection}

Oxford Diffraction Gemini-S CCD-detector diffractometer

Radiation source: Enhance (Mo) X-ray source

Graphite monochromator

Detector resolution: 16.077 pixels $\mathrm{mm}^{-1}$

$\omega$ scans

Absorption correction: multi-scan

(CrysAlis PRO; Agilent, 2013)

$T_{\min }=0.960, T_{\max }=0.980$

\section{Refinement}

Refinement on $F^{2}$

Least-squares matrix: full

$R\left[F^{2}>2 \sigma\left(F^{2}\right)\right]=0.053$

$w R\left(F^{2}\right)=0.116$

$S=1.10$

1738 reflections

118 parameters

0 restraints

Primary atom site location: structure-invariant direct methods
$F(000)=392$

$D_{\mathrm{x}}=1.407 \mathrm{Mg} \mathrm{m}^{-3}$

Mo $K \alpha$ radiation, $\lambda=0.71073 \AA$

Cell parameters from 1311 reflections

$\theta=3.3-28.0^{\circ}$

$\mu=0.12 \mathrm{~mm}^{-1}$

$T=200 \mathrm{~K}$

Plate, colourless

$0.26 \times 0.20 \times 0.05 \mathrm{~mm}$

5619 measured reflections

1738 independent reflections

1304 reflections with $I>2 \sigma(I)$

$R_{\text {int }}=0.033$

$\theta_{\text {max }}=26.0^{\circ}, \theta_{\min }=3.1^{\circ}$

$h=-22 \rightarrow 20$

$k=-8 \rightarrow 8$

$l=-7 \rightarrow 8$

Special details

Secondary atom site location: difference Fourier map

Hydrogen site location: inferred from neighbouring sites

H-atom parameters constrained

$w=1 /\left[\sigma^{2}\left(F_{\mathrm{o}}{ }^{2}\right)+(0.0377 P)^{2}+0.2467 P\right]$

where $P=\left(F_{\mathrm{o}}^{2}+2 F_{\mathrm{c}}^{2}\right) / 3$

$(\Delta / \sigma)_{\max }<0.001$

$\Delta \rho_{\max }=0.16$ e $\AA^{-3}$

$\Delta \rho_{\min }=-0.22$ e $\AA^{-3}$

Geometry. Bond distances, angles etc. have been calculated using the rounded fractional coordinates. All su's are estimated from the variances of the (full) variance-covariance matrix. The cell e.s.d.'s are taken into account in the estimation of distances, angles and torsion angles 
Refinement. Refinement of $F^{2}$ against ALL reflections. The weighted $R$-factor $w R$ and goodness of fit $S$ are based on $F^{2}$, conventional $R$-factors $R$ are based on $F$, with $F$ set to zero for negative $F^{2}$. The threshold expression of $F^{2}>\sigma\left(F^{2}\right)$ is used only for calculating $R$-factors(gt) etc. and is not relevant to the choice of reflections for refinement. $R$-factors based on $F^{2}$ are statistically about twice as large as those based on $F$, and $R$ - factors based on ALL data will be even larger.

Fractional atomic coordinates and isotropic or equivalent isotropic displacement parameters $\left(\AA^{2}\right)$

\begin{tabular}{lllll}
\hline & $x$ & $y$ & $z$ & $U_{\text {iso }} / U_{\text {eq }}$ \\
\hline F4 & $0.97409(8)$ & $0.4959(2)$ & $-0.3100(2)$ & $0.0761(6)$ \\
O11 & $0.70924(8)$ & $0.4850(2)$ & $0.0189(2)$ & $0.0451(5)$ \\
O13 & $0.58131(8)$ & $0.4391(2)$ & $0.1749(2)$ & $0.0439(5)$ \\
O14 & $0.60820(8)$ & $0.6448(2)$ & $0.4167(2)$ & $0.0504(6)$ \\
C1 & $0.77687(12)$ & $0.4984(3)$ & $-0.0575(3)$ & $0.0409(8)$ \\
C2 & $0.78437(13)$ & $0.4048(3)$ & $-0.2361(3)$ & $0.0487(8)$ \\
C3 & $0.85114(14)$ & $0.4052(4)$ & $-0.3198(3)$ & $0.0548(9)$ \\
C4 & $0.90870(13)$ & $0.4967(4)$ & $-0.2263(4)$ & $0.0529(9)$ \\
C5 & $0.90226(13)$ & $0.5918(4)$ & $-0.0520(3)$ & $0.0520(9)$ \\
C6 & $0.83562(12)$ & $0.5925(3)$ & $0.0333(3)$ & $0.0463(8)$ \\
C12 & $0.69955(11)$ & $0.5819(3)$ & $0.2002(3)$ & $0.0411(7)$ \\
C13 & $0.62335(12)$ & $0.5521(3)$ & $0.2684(3)$ & $0.0382(7)$ \\
N1 & $0.43689(9)$ & $0.5487(3)$ & $0.2527(2)$ & $0.0417(6)$ \\
H2 & 0.74400 & 0.34140 & -0.29970 & $0.0580^{*}$ \\
H3 & 0.85700 & 0.34210 & -0.44160 & $0.0660^{*}$ \\
H5 & 0.94280 & 0.65620 & 0.00930 & $0.0620^{*}$ \\
H6 & 0.83020 & 0.65760 & 0.15420 & $0.0560^{*}$ \\
H121 & 0.70810 & 0.71780 & 0.18130 & $0.0490^{*}$ \\
H122 & 0.73570 & 0.53600 & 0.30340 & $0.0490^{*}$ \\
H11 & 0.48210 & 0.50970 & 0.22990 & $0.0500^{*}$ \\
H12 & 0.42870 & 0.67850 & 0.28670 & $0.0500^{*}$ \\
H13 & 0.40960 & 0.51140 & 0.13330 & $0.0500^{*}$ \\
H14 & 0.42010 & 0.48100 & 0.35030 & $0.0500^{*}$ \\
& & & & \\
\hline
\end{tabular}

Atomic displacement parameters $\left(\AA^{2}\right)$

\begin{tabular}{lllllll}
\hline & $U^{11}$ & $U^{22}$ & $U^{33}$ & $U^{12}$ & $U^{13}$ & $U^{23}$ \\
\hline F4 & $0.0554(10)$ & $0.1149(13)$ & $0.0604(10)$ & $0.0161(9)$ & $0.0230(8)$ & $0.0035(9)$ \\
O11 & $0.0373(9)$ & $0.0661(11)$ & $0.0316(8)$ & $0.0035(7)$ & $0.0001(6)$ & $-0.0140(7)$ \\
O13 & $0.0428(9)$ & $0.0527(9)$ & $0.0362(9)$ & $-0.0041(7)$ & $0.0034(7)$ & $-0.0052(7)$ \\
O14 & $0.0585(11)$ & $0.0630(10)$ & $0.0308(9)$ & $-0.0037(8)$ & $0.0115(7)$ & $-0.0097(8)$ \\
C1 & $0.0382(13)$ & $0.0554(14)$ & $0.0291(12)$ & $0.0110(10)$ & $0.0012(9)$ & $-0.0016(10)$ \\
C2 & $0.0517(15)$ & $0.0630(16)$ & $0.0312(12)$ & $0.0072(12)$ & $0.0009(10)$ & $-0.0062(11)$ \\
C3 & $0.0636(17)$ & $0.0717(17)$ & $0.0298(12)$ & $0.0147(14)$ & $0.0085(11)$ & $-0.0051(12)$ \\
C4 & $0.0437(15)$ & $0.0744(17)$ & $0.0416(14)$ & $0.0159(13)$ & $0.0114(11)$ & $0.0054(12)$ \\
C5 & $0.0397(14)$ & $0.0719(17)$ & $0.0441(14)$ & $0.0070(12)$ & $0.0002(11)$ & $0.0013(12)$ \\
C6 & $0.0398(13)$ & $0.0651(16)$ & $0.0338(12)$ & $0.0072(11)$ & $-0.0006(10)$ & $-0.0073(11)$ \\
C12 & $0.0409(13)$ & $0.0565(14)$ & $0.0253(11)$ & $0.0054(11)$ & $-0.0033(9)$ & $-0.0088(10)$ \\
C13 & $0.0432(13)$ & $0.0445(12)$ & $0.0263(11)$ & $0.0034(10)$ & $-0.0019(9)$ & $0.0032(10)$ \\
N1 & $0.0438(11)$ & $0.0555(12)$ & $0.0257(9)$ & $0.0053(9)$ & $0.0024(8)$ & $-0.0060(8)$ \\
& & & & & &
\end{tabular}


Geometric parameters $\left(\AA,{ }^{\circ}\right)$

\begin{tabular}{|c|c|c|c|}
\hline $\mathrm{F} 4-\mathrm{C} 4$ & $1.359(3)$ & $\mathrm{C} 2-\mathrm{C} 3$ & $1.382(3)$ \\
\hline $\mathrm{O} 11-\mathrm{C} 1$ & $1.378(3)$ & $\mathrm{C} 3-\mathrm{C} 4$ & $1.366(4)$ \\
\hline $\mathrm{O} 11-\mathrm{C} 12$ & $1.427(2)$ & $\mathrm{C} 4-\mathrm{C} 5$ & $1.370(4)$ \\
\hline $\mathrm{O} 13-\mathrm{C} 13$ & $1.260(3)$ & $\mathrm{C} 5-\mathrm{C} 6$ & $1.385(3)$ \\
\hline $\mathrm{O} 14-\mathrm{C} 13$ & $1.246(2)$ & $\mathrm{C} 12-\mathrm{C} 13$ & $1.516(3)$ \\
\hline $\mathrm{N} 1-\mathrm{H} 13$ & 0.9600 & $\mathrm{C} 2-\mathrm{H} 2$ & 0.9500 \\
\hline $\mathrm{N} 1-\mathrm{H} 14$ & 0.8900 & $\mathrm{C} 3-\mathrm{H} 3$ & 0.9500 \\
\hline $\mathrm{N} 1-\mathrm{H} 11$ & 0.9000 & $\mathrm{C} 5-\mathrm{H} 5$ & 0.9500 \\
\hline $\mathrm{N} 1-\mathrm{H} 12$ & 0.9700 & $\mathrm{C} 6-\mathrm{H} 6$ & 0.9500 \\
\hline $\mathrm{C} 1-\mathrm{C} 6$ & $1.384(3)$ & $\mathrm{C} 12-\mathrm{H} 121$ & 0.9900 \\
\hline $\mathrm{C} 1-\mathrm{C} 2$ & $1.393(3)$ & $\mathrm{C} 12-\mathrm{H} 122$ & 0.9900 \\
\hline $\mathrm{C} 1-\mathrm{O} 11-\mathrm{C} 12$ & $116.74(16)$ & $\mathrm{O} 11-\mathrm{C} 12-\mathrm{C} 13$ & $111.00(16)$ \\
\hline $\mathrm{H} 12-\mathrm{N} 1-\mathrm{H} 14$ & 106.00 & $\mathrm{O} 13-\mathrm{C} 13-\mathrm{O} 14$ & $125.6(2)$ \\
\hline $\mathrm{H} 13-\mathrm{N} 1-\mathrm{H} 14$ & 107.00 & $\mathrm{O} 14-\mathrm{C} 13-\mathrm{C} 12$ & $115.18(18)$ \\
\hline $\mathrm{H} 11-\mathrm{N} 1-\mathrm{H} 12$ & 120.00 & $\mathrm{O} 13-\mathrm{C} 13-\mathrm{C} 12$ & $119.18(18)$ \\
\hline $\mathrm{H} 11-\mathrm{N} 1-\mathrm{H} 13$ & 102.00 & $\mathrm{C} 1-\mathrm{C} 2-\mathrm{H} 2$ & 120.00 \\
\hline $\mathrm{H} 11-\mathrm{N} 1-\mathrm{H} 14$ & 109.00 & $\mathrm{C} 3-\mathrm{C} 2-\mathrm{H} 2$ & 120.00 \\
\hline $\mathrm{H} 12-\mathrm{N} 1-\mathrm{H} 13$ & 113.00 & $\mathrm{C} 4-\mathrm{C} 3-\mathrm{H} 3$ & 120.00 \\
\hline $\mathrm{O} 11-\mathrm{C} 1-\mathrm{C} 6$ & $124.43(18)$ & $\mathrm{C} 2-\mathrm{C} 3-\mathrm{H} 3$ & 120.00 \\
\hline $\mathrm{C} 2-\mathrm{C} 1-\mathrm{C} 6$ & $120.1(2)$ & $\mathrm{C} 6-\mathrm{C} 5-\mathrm{H} 5$ & 121.00 \\
\hline $\mathrm{O} 11-\mathrm{C} 1-\mathrm{C} 2$ & $115.45(19)$ & $\mathrm{C} 4-\mathrm{C} 5-\mathrm{H} 5$ & 121.00 \\
\hline $\mathrm{C} 1-\mathrm{C} 2-\mathrm{C} 3$ & $119.3(2)$ & $\mathrm{C} 1-\mathrm{C} 6-\mathrm{H} 6$ & 120.00 \\
\hline $\mathrm{C} 2-\mathrm{C} 3-\mathrm{C} 4$ & $119.6(2)$ & $\mathrm{C} 5-\mathrm{C} 6-\mathrm{H} 6$ & 120.00 \\
\hline $\mathrm{C} 3-\mathrm{C} 4-\mathrm{C} 5$ & $122.0(2)$ & $\mathrm{H} 121-\mathrm{C} 12-\mathrm{H} 122$ & 108.00 \\
\hline $\mathrm{F} 4-\mathrm{C} 4-\mathrm{C} 3$ & $119.1(2)$ & $\mathrm{O} 11-\mathrm{C} 12-\mathrm{H} 121$ & 109.00 \\
\hline $\mathrm{F} 4-\mathrm{C} 4-\mathrm{C} 5$ & $118.9(2)$ & $\mathrm{O} 11-\mathrm{C} 12-\mathrm{H} 122$ & 109.00 \\
\hline $\mathrm{C} 4-\mathrm{C} 5-\mathrm{C} 6$ & $118.9(2)$ & $\mathrm{C} 13-\mathrm{C} 12-\mathrm{H} 121$ & 109.00 \\
\hline $\mathrm{C} 1-\mathrm{C} 6-\mathrm{C} 5$ & $120.0(2)$ & $\mathrm{C} 13-\mathrm{C} 12-\mathrm{H} 122$ & 109.00 \\
\hline $\mathrm{C} 12-\mathrm{O} 11-\mathrm{C} 1-\mathrm{C} 2$ & $-179.05(18)$ & $\mathrm{C} 2-\mathrm{C} 3-\mathrm{C} 4-\mathrm{F} 4$ & $179.9(2)$ \\
\hline $\mathrm{C} 12-\mathrm{O} 11-\mathrm{C} 1-\mathrm{C} 6$ & $2.5(3)$ & $\mathrm{C} 2-\mathrm{C} 3-\mathrm{C} 4-\mathrm{C} 5$ & $-1.1(4)$ \\
\hline $\mathrm{C} 1-\mathrm{O} 11-\mathrm{C} 12-\mathrm{C} 13$ & $-178.98(17)$ & $\mathrm{F} 4-\mathrm{C} 4-\mathrm{C} 5-\mathrm{C} 6$ & $-180.0(2)$ \\
\hline $\mathrm{O} 11-\mathrm{C} 1-\mathrm{C} 2-\mathrm{C} 3$ & $-177.7(2)$ & $\mathrm{C} 3-\mathrm{C} 4-\mathrm{C} 5-\mathrm{C} 6$ & $1.0(4)$ \\
\hline $\mathrm{C} 6-\mathrm{C} 1-\mathrm{C} 2-\mathrm{C} 3$ & $0.8(3)$ & $\mathrm{C} 4-\mathrm{C} 5-\mathrm{C} 6-\mathrm{C} 1$ & $-0.1(4)$ \\
\hline $\mathrm{O} 11-\mathrm{C} 1-\mathrm{C} 6-\mathrm{C} 5$ & $177.5(2)$ & $\mathrm{O} 11-\mathrm{C} 12-\mathrm{C} 13-\mathrm{O} 13$ & $6.0(3)$ \\
\hline $\mathrm{C} 2-\mathrm{C} 1-\mathrm{C} 6-\mathrm{C} 5$ & $-0.8(3)$ & $\mathrm{O} 11-\mathrm{C} 12-\mathrm{C} 13-\mathrm{O} 14$ & $-174.13(17)$ \\
\hline $\mathrm{C} 1-\mathrm{C} 2-\mathrm{C} 3-\mathrm{C} 4$ & $0.2(4)$ & & \\
\hline
\end{tabular}

Hydrogen-bond geometry $\left(A,{ }^{\circ}\right)$

\begin{tabular}{lllll}
\hline$D-\mathrm{H} \cdots A$ & $D-\mathrm{H}$ & $\mathrm{H} \cdots A$ & $D \cdots A$ & $D-\mathrm{H} \cdots A$ \\
\hline $\mathrm{N} 1-\mathrm{H} 11 \cdots \mathrm{O} 13$ & 0.90 & 1.95 & $2.847(2)$ & 177 \\
$\mathrm{~N} 1-\mathrm{H} 11 \cdots \mathrm{O} 14$ & 0.90 & 2.55 & $3.347(2)$ & 135 \\
$\mathrm{~N} 1-\mathrm{H} 12 \cdots \mathrm{O} 13^{\mathrm{i}}$ & 0.97 & 1.88 & $2.847(3)$ & 173 \\
$\mathrm{~N} 1-\mathrm{H} 13 \cdots \mathrm{O} 11^{\mathrm{ii}}$ & 0.96 & 2.36 & $3.172(2)$ & 142
\end{tabular}




\begin{tabular}{lllll}
$\mathrm{N} 1-\mathrm{H} 13 \cdots \mathrm{O} 13^{\mathrm{ii}}$ & 0.96 & 2.13 & $2.892(2)$ & 135 \\
$\mathrm{~N} 1-\mathrm{H} 14 \cdots \mathrm{O} 14^{\mathrm{iii}}$ & 0.89 & 1.91 & $2.793(2)$ & 173 \\
\hline
\end{tabular}

Symmetry codes: (i) $-x+1, y+1 / 2,-z+1 / 2$; (ii) $-x+1,-y+1,-z$; (iii) $-x+1,-y+1,-z+1$.

\section{(III) Ammonium (4-chloro-2-methylphenoxy)acetate hemihydrate}

\section{Crystal data}

$\mathrm{NH}_{4}^{+} \cdot \mathrm{C}_{9} \mathrm{H}_{8} \mathrm{ClNO}_{3}{ }^{-} \cdot 0.5 \mathrm{H}_{2} \mathrm{O}$

$M_{r}=226.65$

Monoclinic, $C 2 / c$

Hall symbol: $-\mathrm{C} 2 \mathrm{yc}$

$a=38.0396$ (9) $\AA$

$b=4.4560(8) \AA$

$c=12.944(5) \AA$

$\beta=104.575(5)^{\circ}$

$V=2123.5(9) \AA^{3}$

$Z=8$

\section{Data collection}

Oxford Diffraction Gemini-S CCD-detector diffractometer

Radiation source: Enhance (Mo) X-ray source

Graphite monochromator

Detector resolution: 16.077 pixels $\mathrm{mm}^{-1}$

$\omega$ scans

Absorption correction: multi-scan

(CrysAlis PRO; Agilent, 2013)

$T_{\min }=0.913, T_{\max }=0.980$

Refinement

Refinement on $F^{2}$

Least-squares matrix: full

$R\left[F^{2}>2 \sigma\left(F^{2}\right)\right]=0.036$

$w R\left(F^{2}\right)=0.091$

$S=1.03$

2087 reflections

132 parameters

0 restraints

Primary atom site location: structure-invariant direct methods
$F(000)=952$
$D_{\mathrm{x}}=1.418 \mathrm{Mg} \mathrm{m}^{-3}$

Mo $K \alpha$ radiation, $\lambda=0.71073 \AA$

Cell parameters from 1819 reflections

$\theta=4.4-28.1^{\circ}$

$\mu=0.35 \mathrm{~mm}^{-1}$

$T=200 \mathrm{~K}$

Plate, colourless

$0.35 \times 0.35 \times 0.10 \mathrm{~mm}$

6215 measured reflections

2087 independent reflections

1771 reflections with $I>2 \sigma(I)$

$R_{\text {int }}=0.030$

$\theta_{\text {max }}=26.0^{\circ}, \theta_{\text {min }}=3.2^{\circ}$

$h=-46 \rightarrow 46$

$k=-5 \rightarrow 5$

$l=-15 \rightarrow 15$

Special details

Geometry. Bond distances, angles etc. have been calculated using the rounded fractional coordinates. All su's are estimated from the variances of the (full) variance-covariance matrix. The cell e.s.d.'s are taken into account in the estimation of distances, angles and torsion angles

Refinement. Refinement of $F^{2}$ against ALL reflections. The weighted $R$-factor $w R$ and goodness of fit $S$ are based on $F^{2}$, conventional $R$-factors $R$ are based on $F$, with $F$ set to zero for negative $F^{2}$. The threshold expression of $F^{2}>\sigma\left(F^{2}\right)$ is used only for calculating $R$-factors(gt) etc. and is not relevant to the choice of reflections for refinement. $R$-factors based on $F^{2}$ are statistically about twice as large as those based on $F$, and $R$ - factors based on ALL data will be even larger.

Fractional atomic coordinates and isotropic or equivalent isotropic displacement parameters $\left(\AA^{2}\right)$

\begin{tabular}{llllll}
\hline & $x$ & $y$ & $z$ & $U_{\text {iss }} / U_{\text {eq }}$ & Occ. $(<1)$ \\
\hline C14 & $0.24818(1)$ & $0.32330(12)$ & $0.36021(4)$ & $0.0372(2)$ &
\end{tabular}




\begin{tabular}{|c|c|c|c|c|c|}
\hline O11 & $0.39197(3)$ & $0.8996(3)$ & $0.46961(9)$ & $0.0262(4)$ & \\
\hline $\mathrm{O} 13$ & $0.45425(3)$ & $1.2161(3)$ & $0.49906(10)$ & $0.0296(4)$ & \\
\hline $\mathrm{O} 14$ & $0.44789(4)$ & $1.4021(3)$ & $0.65332(10)$ & $0.0339(4)$ & \\
\hline $\mathrm{C} 1$ & $0.35808(5)$ & $0.7686(4)$ & $0.44981(13)$ & $0.0224(5)$ & \\
\hline $\mathrm{C} 2$ & $0.34876(5)$ & $0.5869(4)$ & $0.35836(13)$ & $0.0246(5)$ & \\
\hline $\mathrm{C} 3$ & $0.31491(5)$ & $0.4496(4)$ & $0.33324(14)$ & $0.0269(5)$ & \\
\hline $\mathrm{C} 4$ & $0.29091(5)$ & $0.4918(4)$ & $0.39679(14)$ & 0.0264 & \\
\hline $\mathrm{C} 5$ & $0.30027(5)$ & $0.6675(4)$ & $0.48684(14)$ & $0.0275(6)$ & \\
\hline $\mathrm{C} 6$ & $0.33390(5)$ & $0.8070(4)$ & $0.51321(14)$ & $0.0258(5)$ & \\
\hline $\mathrm{C} 12$ & $0.40205(5)$ & $1.0812(4)$ & $0.56298(14)$ & $0.0242(5)$ & \\
\hline $\mathrm{C} 13$ & $0.43762(5)$ & $1.2444(4)$ & $0.57156(14)$ & $0.0234(5)$ & \\
\hline $\mathrm{C} 21$ & $0.37517(5)$ & $0.5430(5)$ & $0.29002(15)$ & $0.0370(6)$ & \\
\hline $\mathrm{O} 1 \mathrm{~W}$ & 0.50000 & $1.8306(4)$ & 0.75000 & $0.0464(7)$ & \\
\hline $\mathrm{N} 1$ & $0.46781(3)$ & $0.7274(3)$ & $0.37900(10)$ & $0.0156(4)$ & \\
\hline $\mathrm{H} 3$ & 0.30820 & 0.32800 & 0.27310 & $0.0320^{*}$ & \\
\hline H5 & 0.28420 & 0.69260 & 0.52960 & $0.0330^{*}$ & \\
\hline H6 & 0.34030 & 0.92710 & 0.57380 & $0.0310^{*}$ & \\
\hline $\mathrm{H} 121$ & 0.40420 & 0.95550 & 0.62550 & $0.0290^{*}$ & \\
\hline H122 & 0.38300 & 1.22720 & 0.56190 & $0.0290 *$ & \\
\hline $\mathrm{H} 211$ & 0.39700 & 0.65450 & 0.31980 & $0.0550^{*}$ & 0.500 \\
\hline $\mathrm{H} 212$ & 0.36440 & 0.61300 & 0.21890 & $0.0550^{*}$ & 0.500 \\
\hline $\mathrm{H} 213$ & 0.38090 & 0.33380 & 0.28790 & $0.0550^{*}$ & 0.500 \\
\hline $\mathrm{H} 214$ & 0.36460 & 0.41300 & 0.23130 & $0.0550^{*}$ & 0.500 \\
\hline $\mathrm{H} 215$ & 0.39710 & 0.45450 & 0.33210 & $0.0550^{*}$ & 0.500 \\
\hline $\mathrm{H} 216$ & 0.38060 & 0.73370 & 0.26320 & $0.0550 *$ & 0.500 \\
\hline $\mathrm{H} 11 \mathrm{~W}$ & 0.48390 & 1.71260 & 0.70860 & $0.0700^{*}$ & \\
\hline H11 & 0.49010 & 0.73870 & 0.39810 & $0.0190^{*}$ & \\
\hline $\mathrm{H} 12$ & 0.45970 & 0.71470 & 0.31450 & $0.0190 *$ & \\
\hline H13 & 0.46250 & 0.57360 & 0.40950 & $0.0190 *$ & \\
\hline H14 & 0.45930 & 0.86670 & 0.40680 & $0.0190 *$ & \\
\hline
\end{tabular}

Atomic displacement parameters $\left(\AA^{2}\right)$

\begin{tabular}{lllllll}
\hline & $U^{11}$ & $U^{22}$ & $U^{33}$ & $U^{12}$ & $U^{13}$ & $U^{23}$ \\
\hline C14 & $0.0264(3)$ & $0.0466(3)$ & $0.0378(3)$ & $-0.0121(2)$ & $0.0064(2)$ & $-0.0044(2)$ \\
O11 & $0.0233(7)$ & $0.0335(7)$ & $0.0230(6)$ & $-0.0071(5)$ & $0.0083(5)$ & $-0.0075(5)$ \\
O13 & $0.0293(7)$ & $0.0279(6)$ & $0.0355(7)$ & $-0.0036(6)$ & $0.0155(6)$ & $-0.0015(6)$ \\
O14 & $0.0340(8)$ & $0.0378(7)$ & $0.0268(7)$ & $-0.0094(6)$ & $0.0020(6)$ & $-0.0054(6)$ \\
C1 & $0.0215(9)$ & $0.0222(8)$ & $0.0224(9)$ & $-0.0010(7)$ & $0.0037(7)$ & $0.0028(7)$ \\
C2 & $0.0253(9)$ & $0.0277(9)$ & $0.0198(8)$ & $0.0005(7)$ & $0.0041(7)$ & $0.0002(7)$ \\
C3 & $0.0278(10)$ & $0.0287(9)$ & $0.0223(9)$ & $-0.0021(8)$ & $0.0027(8)$ & $-0.0023(8)$ \\
C4 & $0.0217(9)$ & $0.0277(9)$ & $0.0277(9)$ & $-0.0031(7)$ & $0.0023(8)$ & $0.0037(8)$ \\
C5 & $0.0230(10)$ & $0.0332(10)$ & $0.0282(9)$ & $0.0003(8)$ & $0.0099(8)$ & $0.0017(8)$ \\
C6 & $0.0259(10)$ & $0.0281(9)$ & $0.0234(9)$ & $-0.0011(8)$ & $0.0062(7)$ & $-0.0022(8)$ \\
C12 & $0.0244(9)$ & $0.0276(9)$ & $0.0208(8)$ & $-0.0026(7)$ & $0.0059(7)$ & $-0.0033(7)$ \\
C13 & $0.0247(9)$ & $0.0215(8)$ & $0.0232(9)$ & $0.0029(7)$ & $0.0045(7)$ & $0.0042(7)$ \\
C21 & $0.0331(11)$ & $0.0520(12)$ & $0.0278(10)$ & $-0.0083(10)$ & $0.0114(9)$ & $-0.0126(9)$ \\
O1W & $0.0421(13)$ & $0.0259(10)$ & $0.0667(15)$ & 0.0000 & $0.0053(11)$ & 0.0000
\end{tabular}




$\mathrm{N} 1 \quad 0.0141(7) \quad 0.0159(6) \quad 0.0179(6) \quad-0.0006(5) \quad 0.0060(5) \quad-0.0004(5)$

Geometric parameters $\left(\AA,{ }^{\circ}\right)$

\begin{tabular}{|c|c|c|c|}
\hline $\mathrm{Cl} 4-\mathrm{C} 4$ & $1.744(3)$ & $\mathrm{C} 3-\mathrm{C} 4$ & $1.387(3)$ \\
\hline $\mathrm{O} 11-\mathrm{C} 1$ & $1.379(3)$ & $\mathrm{C} 4-\mathrm{C} 5$ & $1.375(3)$ \\
\hline $\mathrm{O} 11-\mathrm{C} 12$ & $1.425(3)$ & $\mathrm{C} 5-\mathrm{C} 6$ & $1.386(3)$ \\
\hline $\mathrm{O} 13-\mathrm{C} 13$ & $1.263(3)$ & $\mathrm{C} 12-\mathrm{C} 13$ & $1.515(3)$ \\
\hline $\mathrm{O} 14-\mathrm{C} 13$ & $1.248(3)$ & $\mathrm{C} 3-\mathrm{H} 3$ & 0.9300 \\
\hline $\mathrm{O} 1 \mathrm{~W}-\mathrm{H} 11 \mathrm{~W}$ & 0.8800 & $\mathrm{C} 5-\mathrm{H} 5$ & 0.9300 \\
\hline $\mathrm{O} 1 \mathrm{~W}-\mathrm{H} 11 \mathrm{~W}^{\mathrm{i}}$ & 0.8800 & $\mathrm{C} 6-\mathrm{H} 6$ & 0.9300 \\
\hline $\mathrm{N} 1-\mathrm{H} 12$ & 0.8200 & $\mathrm{C} 12-\mathrm{H} 121$ & 0.9700 \\
\hline N1-H11 & 0.8200 & $\mathrm{C} 12-\mathrm{H} 122$ & 0.9700 \\
\hline $\mathrm{N} 1-\mathrm{H} 13$ & 0.8400 & $\mathrm{C} 21-\mathrm{H} 216$ & 0.9600 \\
\hline $\mathrm{N} 1-\mathrm{H} 14$ & 0.8200 & $\mathrm{C} 21-\mathrm{H} 211$ & 0.9600 \\
\hline $\mathrm{C} 1-\mathrm{C} 2$ & $1.404(3)$ & $\mathrm{C} 21-\mathrm{H} 212$ & 0.9600 \\
\hline $\mathrm{C} 1-\mathrm{C} 6$ & $1.389(3)$ & $\mathrm{C} 21-\mathrm{H} 213$ & 0.9600 \\
\hline $\mathrm{C} 2-\mathrm{C} 21$ & $1.509(3)$ & $\mathrm{C} 21-\mathrm{H} 214$ & 0.9600 \\
\hline $\mathrm{C} 2-\mathrm{C} 3$ & $1.388(3)$ & $\mathrm{C} 21-\mathrm{H} 215$ & 0.9600 \\
\hline $\mathrm{C} 1-\mathrm{O} 11-\mathrm{C} 12$ & $115.95(13)$ & $\mathrm{C} 2-\mathrm{C} 3-\mathrm{H} 3$ & 120.00 \\
\hline $\mathrm{H} 11 \mathrm{~W}-\mathrm{O} 1 \mathrm{~W}-\mathrm{H} 11 \mathrm{~W}^{\mathrm{i}}$ & 107.00 & $\mathrm{C} 4-\mathrm{C} 3-\mathrm{H} 3$ & 120.00 \\
\hline $\mathrm{H} 12-\mathrm{N} 1-\mathrm{H} 14$ & 114.00 & $\mathrm{C} 4-\mathrm{C} 5-\mathrm{H} 5$ & 120.00 \\
\hline H13-N1-H14 & 104.00 & $\mathrm{C} 6-\mathrm{C} 5-\mathrm{H} 5$ & 120.00 \\
\hline $\mathrm{H} 11-\mathrm{N} 1-\mathrm{H} 12$ & 114.00 & $\mathrm{C} 5-\mathrm{C} 6-\mathrm{H} 6$ & 120.00 \\
\hline $\mathrm{H} 11-\mathrm{N} 1-\mathrm{H} 13$ & 105.00 & $\mathrm{C} 1-\mathrm{C} 6-\mathrm{H} 6$ & 120.00 \\
\hline $\mathrm{H} 11-\mathrm{N} 1-\mathrm{H} 14$ & 108.00 & $\mathrm{C} 13-\mathrm{C} 12-\mathrm{H} 122$ & 109.00 \\
\hline $\mathrm{H} 12-\mathrm{N} 1-\mathrm{H} 13$ & 111.00 & $\mathrm{C} 13-\mathrm{C} 12-\mathrm{H} 121$ & 109.00 \\
\hline $\mathrm{O} 11-\mathrm{C} 1-\mathrm{C} 2$ & $115.26(16)$ & $\mathrm{O} 11-\mathrm{C} 12-\mathrm{H} 121$ & 109.00 \\
\hline $\mathrm{O} 11-\mathrm{C} 1-\mathrm{C} 6$ & $124.41(15)$ & $\mathrm{O} 11-\mathrm{C} 12-\mathrm{H} 122$ & 109.00 \\
\hline $\mathrm{C} 2-\mathrm{C} 1-\mathrm{C} 6$ & $120.33(17)$ & $\mathrm{H} 121-\mathrm{C} 12-\mathrm{H} 122$ & 108.00 \\
\hline $\mathrm{C} 1-\mathrm{C} 2-\mathrm{C} 21$ & $120.32(17)$ & $\mathrm{C} 2-\mathrm{C} 21-\mathrm{H} 211$ & 109.00 \\
\hline $\mathrm{C} 1-\mathrm{C} 2-\mathrm{C} 3$ & $118.30(17)$ & $\mathrm{C} 2-\mathrm{C} 21-\mathrm{H} 212$ & 109.00 \\
\hline $\mathrm{C} 3-\mathrm{C} 2-\mathrm{C} 21$ & $121.37(16)$ & $\mathrm{C} 2-\mathrm{C} 21-\mathrm{H} 213$ & 109.00 \\
\hline $\mathrm{C} 2-\mathrm{C} 3-\mathrm{C} 4$ & $120.77(16)$ & $\mathrm{C} 2-\mathrm{C} 21-\mathrm{H} 214$ & 110.00 \\
\hline $\mathrm{C} 3-\mathrm{C} 4-\mathrm{C} 5$ & $120.76(18)$ & $\mathrm{C} 2-\mathrm{C} 21-\mathrm{H} 215$ & 109.00 \\
\hline $\mathrm{Cl} 4-\mathrm{C} 4-\mathrm{C} 3$ & $119.22(14)$ & $\mathrm{C} 2-\mathrm{C} 21-\mathrm{H} 216$ & 109.00 \\
\hline $\mathrm{Cl} 4-\mathrm{C} 4-\mathrm{C} 5$ & $120.01(15)$ & $\mathrm{H} 214-\mathrm{C} 21-\mathrm{H} 215$ & 109.00 \\
\hline $\mathrm{C} 4-\mathrm{C} 5-\mathrm{C} 6$ & $119.37(17)$ & $\mathrm{H} 214-\mathrm{C} 21-\mathrm{H} 216$ & 109.00 \\
\hline $\mathrm{C} 1-\mathrm{C} 6-\mathrm{C} 5$ & $120.46(16)$ & $\mathrm{H} 215-\mathrm{C} 21-\mathrm{H} 216$ & 110.00 \\
\hline $\mathrm{O} 11-\mathrm{C} 12-\mathrm{C} 13$ & $112.31(15)$ & $\mathrm{H} 211-\mathrm{C} 21-\mathrm{H} 212$ & 109.00 \\
\hline $\mathrm{O} 13-\mathrm{C} 13-\mathrm{O} 14$ & $125.29(18)$ & $\mathrm{H} 211-\mathrm{C} 21-\mathrm{H} 213$ & 110.00 \\
\hline $\mathrm{O} 13-\mathrm{C} 13-\mathrm{C} 12$ & $120.17(16)$ & $\mathrm{H} 212-\mathrm{C} 21-\mathrm{H} 213$ & 109.00 \\
\hline $\mathrm{O} 14-\mathrm{C} 13-\mathrm{C} 12$ & $114.55(16)$ & & \\
\hline $\mathrm{C} 12-\mathrm{O} 11-\mathrm{C} 1-\mathrm{C} 2$ & $-179.13(15)$ & $\mathrm{C} 1-\mathrm{C} 2-\mathrm{C} 3-\mathrm{C} 4$ & $0.2(3)$ \\
\hline $\mathrm{C} 12-\mathrm{O} 11-\mathrm{C} 1-\mathrm{C} 6$ & $1.0(2)$ & $\mathrm{C} 21-\mathrm{C} 2-\mathrm{C} 3-\mathrm{C} 4$ & $179.98(17)$ \\
\hline $\mathrm{C} 1-\mathrm{O} 11-\mathrm{C} 12-\mathrm{C} 13$ & $-173.34(14)$ & $\mathrm{C} 2-\mathrm{C} 3-\mathrm{C} 4-\mathrm{Cl} 4$ & $178.25(14)$ \\
\hline
\end{tabular}




$\begin{array}{ll}\mathrm{O} 11-\mathrm{C} 1-\mathrm{C} 2-\mathrm{C} 3 & -179.57(15) \\ \mathrm{O} 11-\mathrm{C} 1-\mathrm{C} 2-\mathrm{C} 21 & 0.6(2) \\ \mathrm{C} 6-\mathrm{C} 1-\mathrm{C} 2-\mathrm{C} 3 & 0.3(3) \\ \mathrm{C} 6-\mathrm{C} 1-\mathrm{C} 2-\mathrm{C} 21 & -179.46(17) \\ \mathrm{O} 11-\mathrm{C} 1-\mathrm{C} 6-\mathrm{C} 5 & 179.67(16) \\ \mathrm{C} 2-\mathrm{C} 1-\mathrm{C} 6-\mathrm{C} 5 & -0.2(3)\end{array}$

$\mathrm{C} 2-\mathrm{C} 3-\mathrm{C} 4-\mathrm{C} 5$

$\mathrm{C} 14-\mathrm{C} 4-\mathrm{C} 5-\mathrm{C} 6$

$\mathrm{C} 3-\mathrm{C} 4-\mathrm{C} 5-\mathrm{C} 6$

$\mathrm{C} 4-\mathrm{C} 5-\mathrm{C} 6-\mathrm{C} 1$

$\mathrm{O} 11-\mathrm{C} 12-\mathrm{C} 13-\mathrm{O} 13$

$\mathrm{O} 11-\mathrm{C} 12-\mathrm{C} 13-\mathrm{O} 14$
$-0.8(3)$

$-178.13(14)$

0.9 (3)

$-0.4(3)$

$1.7(2)$

$-178.71(15)$

Symmetry code: (i) $-x+1, y,-z+3 / 2$.

Hydrogen-bond geometry $\left(A,{ }^{\circ}\right)$

\begin{tabular}{lllll}
\hline$D-\mathrm{H} \cdots A$ & $D-\mathrm{H}$ & $\mathrm{H} \cdots A$ & $D \cdots A$ & $D-\mathrm{H} \cdots A$ \\
\hline $\mathrm{N} 1-\mathrm{H} 11 \cdots \mathrm{O} 13^{\mathrm{ii}}$ & 0.82 & 2.21 & $2.998(4)$ & 161 \\
$\mathrm{~N} 1-\mathrm{H} 12 \cdots \mathrm{O} 14^{\mathrm{iii}}$ & 0.82 & 2.09 & $2.886(4)$ & 166 \\
$\mathrm{~N} 1-\mathrm{H} 13 \cdots \mathrm{O} 13^{\text {iv }}$ & 0.84 & 2.04 & $2.877(4)$ & 173 \\
$\mathrm{~N} 1-\mathrm{H} 14 \cdots \mathrm{O} 13$ & 0.82 & 2.00 & $2.798(4)$ & 163 \\
$\mathrm{O} 1 W-\mathrm{H} 11 W \cdots \mathrm{O} 14$ & 0.88 & 1.95 & $2.809(4)$ & 165
\end{tabular}

Symmetry codes: (ii) $-x+1,-y+2,-z+1$; (iii) $x,-y+2, z-1 / 2$; (iv) $x, y-1, z$. 